\title{
Image Matching Based on Local Phase Quantization Applied for Measuring the Tensile Properties of High Elongation Materials
}

\author{
Zhenjun Zhang, ${ }^{1,2}$ Fengji Li, ${ }^{1}$ Min Liu, ${ }^{1}$ and Pawan Kumar Yadav ${ }^{1}$ \\ ${ }^{1}$ Department of Electric and Information Engineering, Hunan University, Yuelu District, Changsha 410082, China \\ ${ }^{2}$ National Engineering Laboratory for Robot Visual Perception and Control Technology, Yuelu District, Changsha 410082, China \\ Correspondence should be addressed to Zhenjun Zhang; zhenjun@hnu.edu.cn
}

Received 10 May 2016; Revised 23 August 2016; Accepted 24 August 2016

Academic Editor: Eric Florentin

Copyright (c) 2016 Zhenjun Zhang et al. This is an open access article distributed under the Creative Commons Attribution License, which permits unrestricted use, distribution, and reproduction in any medium, provided the original work is properly cited.

\begin{abstract}
In recent years, high elongation materials are widely used. Therefore, it is important to investigate the tensile properties of high elongation materials for engineering applications. Video extensometer is equipment for measuring the materials' tensile properties. It uses image processing technology to match data points and measures the actual deformation. However, when measuring high elongation materials, motion blur will appear on the collected images, which can affect the accuracy of image matching. In this paper, we proposed an image matching method which is based on Local Phase Quantization (LPQ) features to reduce the interference of the motion blur and improve the accuracy of the image matching algorithms as well. The experimental results on simulations show that the proposed initialization method is sufficiently accurate to enable the correct convergence of the subsequent optimization in the presence of motion blur. The experiment of uniaxial tensile also verifies the accuracy and robustness of the method.
\end{abstract}

\section{Introduction}

High elongation materials are an important class of materials for structural applications such as transportation, civil infrastructures, and biomedical applications. In actual service conditions, these materials are often subject to both mechanical and environmental loads. These factors will change the material properties and thus have a great influence on the service life and safety performance of these materials. In order to study these factors, the tensile mechanical test should be carried out on these materials.

At present, the most commonly used equipment for the tensile mechanical test is the mechanical extensometer and video extensometer. For the high elongation materials, the mechanical extensometer which is mounted directly onto the material via blade causes many problems such as the following: (1) mutual friction will reduce the measurement accuracy; (2) the total deformation cannot be easily measured in the uniaxial tensile test; (3) the measuring range is limited. Compared with mechanical extensometer, the video extensometer has the following advantages over mechanical extensometer: (1) it has no abrasion on the material; (2) it is applied for different types of specimen and material properties; (3) its measuring range is not limited; (4) it has high precision [1].

Compared with mechanical extensometer, video extensometer has obvious advantages in engineering applications. However, if higher accuracy is pursued, some influence factors can not be ignored, such as out-of-plane displacement, self-heating of the camera, lens distortion, and image blur induced by motion. Reference [2] theoretically describes the measurement errors caused by out-of-plane displacement and self-heating of the camera; it further establishes a highaccuracy two-dimensional digital image correlation (2DDIC) system using a bilateral telecentric lens to minimize the errors. Reference [3] investigates the systematic errors due to lens distortion using the radial lens distortion model and in-plane translation tests; it finds out that the displacement and strain errors at an interrogated image point not only are linear proportion to the distortion coefficient of the camera lens used but also depend on the distance relative to distortion center and its magnitude of the displacement; the paper also proposes a linear least-squares algorithm to estimate the distortion coefficients and then to eliminate 


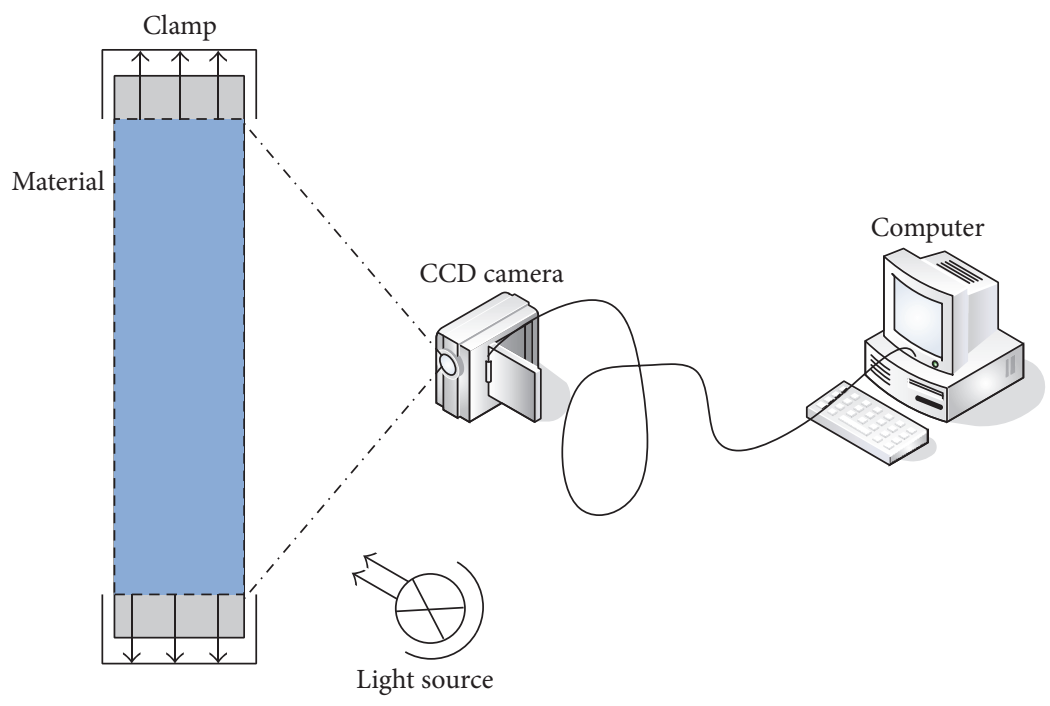

FIGURE 1: Measurement system overview.

the errors. Reference [4] proposes an off-axis digital image correlation method for real-time, noncontact, and targetless measurement of vertical deflection of bridges to achieve subpixel accuracy.

Despite these advances, few works about eliminating extensometer's measurement errors caused by motioninduced image blur to improve the accuracy have been reported. In this paper, we will propose an image matching method for video extensometer to measure the parameters by utilizing Local Phase Quantization (LPQ) feature. This method is robust and performs well on images with serious motion blur and deformation.

The rest of the paper is organized as follows. The basic principle of video extensometer is described in Section 2. The generation mechanism of motion blur is introduced in Section 3. The proposed matching method using Local Phase Quantization is discussed in detail in Section 4. Experimental results on both simulated and real-world data, as well as comparative results with the existing method are presented in Section 5. Finally, Section 6 gives the conclusion.

\section{System Overview}

A schematic diagram of the video extensometer system is shown in Figure 1. The main parts of the video extensometer system are a light source, tensile equipment, CCD camera, and $\mathrm{PC}$ with digital image processing software.

For measurement, first, make the spackle pattern on the material surface, keep the CCD camera's optical axis vertical to the test specimen surface, choose the appropriate focal length, and make sure the field of view covers the whole material. In the tensile stress test, the position of the data point will be changed before and after the deformation. Through the deformation images collected by CCD camera at different times, we can calculate the materials' tensile properties.
2.1. Digital Image Correlation. The video extensometer uses DIC to analyze materials tensile properties [2-4]. The principle of DIC is tracking the same point in undeformed and deformed image and then yields the displacements of the data point. The information contained by the data point cannot identify the same point in a series of deformed images. So we use a square subset centered on the data point to replace the data point, as shown in Figure 2(a). A small square speckle subset of $2 \mathrm{~N} \times 2 \mathrm{~N}$ pixel centered at the data point $P$ in the undeformed image is defined as the reference subset. At the same time, a bigger subset of $2 M \times 2 M$ pixels centered at the corresponding data point $P^{\prime}$ in the deformed image is defined as the searching subset. To calculate the correlation coefficient, the reference subset is moved through the searching subset pixel by pixel. The peak position of the correlation coefficient is the target subset, as can be seen from Figure 2(b).

As shown in Figure 2(b), the stress on the material will cause rigid body displacement, scale distortion, and rotation deformation. So, the intensity of an arbitrary point in the undeformed subset region and the corresponding point in the deformation subset region is related as follows:

$$
g\left(x_{i}^{\prime}, y_{i}^{\prime}\right)=F\left(f\left(x_{i}, y_{i}\right)\right) .
$$

For the shape function $F$, the most widely used function is first-order shape function [5]:

$$
\begin{aligned}
& x^{\prime}=x+u+\frac{\partial u}{\partial x} \Delta x+\frac{\partial u}{\partial y} \Delta y, \\
& y^{\prime}=y+v+\frac{\partial v}{\partial x} \Delta x+\frac{\partial v}{\partial y} \Delta y .
\end{aligned}
$$

The corresponding matrix form is

$$
\left[\begin{array}{l}
X^{\prime} \\
Y^{\prime}
\end{array}\right]=\left[\begin{array}{l}
x \\
y
\end{array}\right]+\left[\begin{array}{lll}
u & u_{x} & u_{y} \\
v & v_{x} & v_{y}
\end{array}\right]\left[\begin{array}{c}
1 \\
\Delta x \\
\Delta y
\end{array}\right],
$$




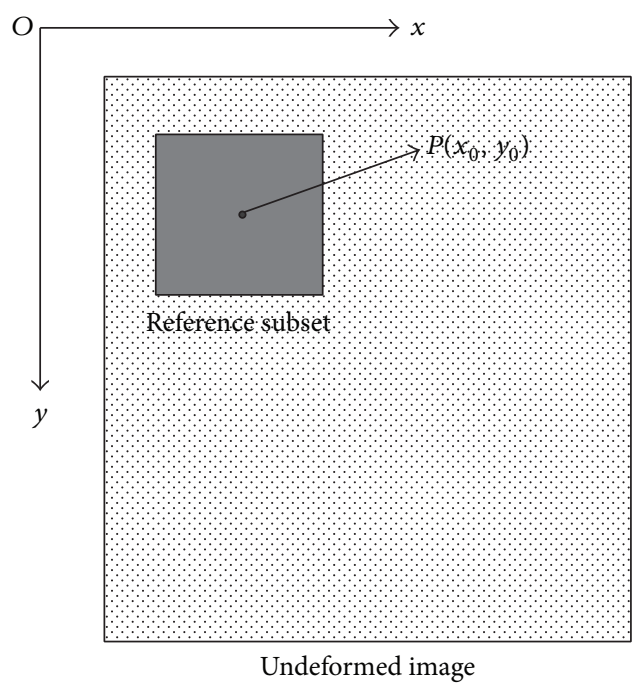

(a)

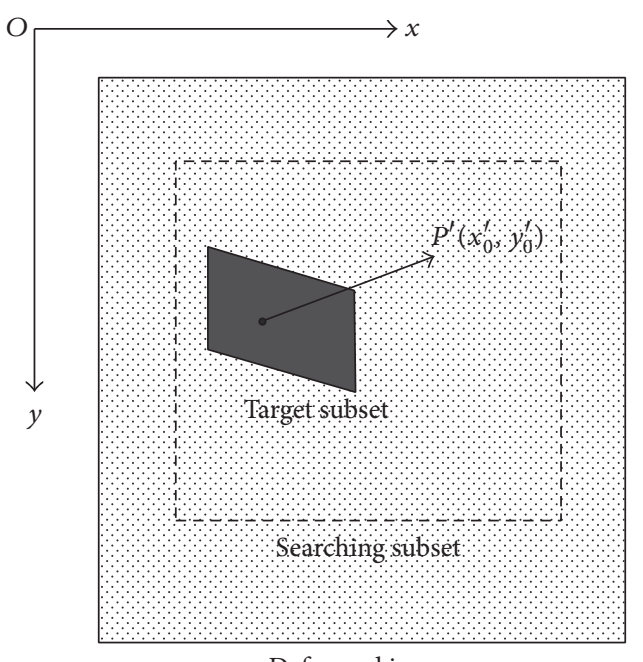

Deformed image

(b)

Figure 2: Procedures of DIC.

where $\partial u / \partial x, \partial u / \partial y, \partial v / \partial x$, and $\partial v / \partial y$ are the displacement gradients of the subset. $\Delta x$ and $\Delta y$ are the distances between point $(x, y)$ and point $\left(x_{0}, y_{0}\right)$, respectively.

Images are obtained in discrete form and intensity values are recorded as "pixels." But the integer pixel locations do not satisfy the accuracy of extensometer. In order to improve the precision of video extensometer, we use subpixel displacement measurement algorithm to get the subpixel displacement. The correlation coefficient curve fitting method, iterative algorithm, and gradient-based algorithm are the three most common methods $[6,7]$. In this paper, we used the N-R iteration algorithm [8] as the subpixel displacement measurement algorithm.

The displacement refined by optimizing correlation using $\mathrm{N}-\mathrm{R}$ iteration algorithm needs to calculate the correlation coefficient which describes the similarity between the reference subset and the target subset. In the actual situation, the spackle pattern will be affected by the lighting condition and the deformation. Tong [9] used different correlation coefficient to analyze the result of DIC and point out the ZNSSD and ZNCC have better robustness and reliability. The relationship between two related functions is as follows:

$$
C_{\mathrm{ZNSSD}}=2\left(1-C_{\mathrm{ZNCC}}\right)
$$

and it is easier to optimize ZNSSD which is expressed as the sum of squares of nonlinear functions:

$$
\begin{aligned}
& C_{\mathrm{ZNSSD}} \\
& =\sum_{i=-M}^{M} \sum_{j=-M}^{M}\left\{\frac{\left[f\left(x_{i}, y_{j}\right)-f_{m}\right]}{\Delta f}-\frac{\left[g\left(x_{i}^{\prime}, y_{j}^{\prime}\right)-g_{m}\right]}{\Delta g}\right\}^{2},
\end{aligned}
$$

where

$$
f_{m}=\frac{1}{(2 M+1)^{2}} \sum_{i=-M}^{M} \sum_{j=-M}^{M} f\left(x_{i}, y_{j}\right),
$$

$$
\begin{aligned}
& g_{m}=\frac{1}{(2 M+1)^{2}} \sum_{i=-M}^{M} \sum_{j=-M}^{M} g\left(x_{i}^{\prime}, y_{j}\right), \\
& \Delta f=\sqrt{\sum_{i=-M}^{M} \sum_{j=-M}^{M}\left[f\left(x_{i}, y_{j}\right)-f_{m}\right]^{2}}, \\
& \Delta g=\sqrt{\sum_{i=-M}^{M} \sum_{j=-M}^{M}\left[g\left(x_{i}^{\prime}, y_{j}^{\prime}\right)-g_{m}\right]^{2}} .
\end{aligned}
$$

Finally, use the optimal parameter which minimizes ZNSSD to calculate the data point displacement and get the materials tensile properties.

\section{Motion Blur}

When the CCD camera collects an image, the image may represent the scene over a period of time, known as the exposure time. But the relative motion between the camera and material during exposure time may result in a blurring image which is motion blur.

The ideal lens imaging model is shown in Figure 3, according to Gaussian lens law:

$$
\frac{1}{u}+\frac{1}{v}=\frac{1}{f},
$$

where $u$ is the object distance, $v$ is image distance, and $f$ is the focal length. Using (7), we can calculate image distance as follows:

$$
v=\frac{f u}{u-f} .
$$




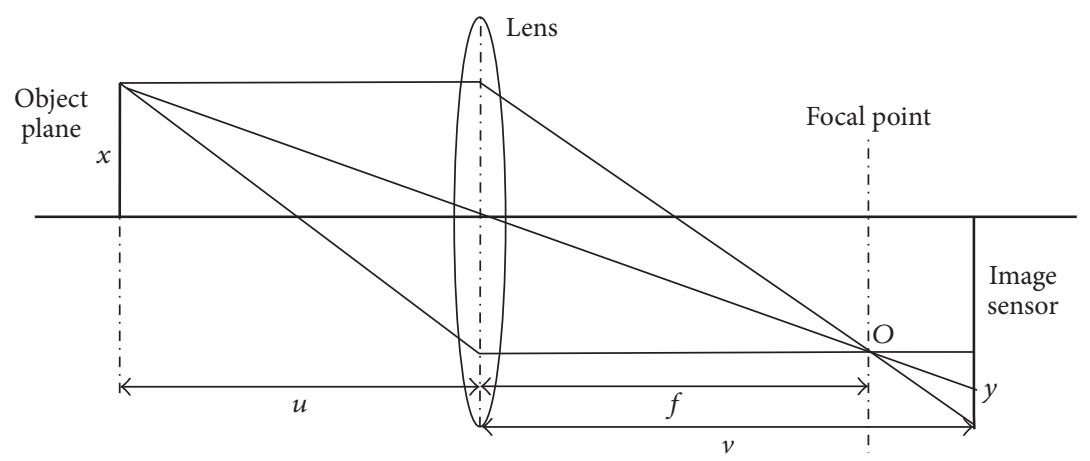

Figure 3: Ideal lens imaging model.

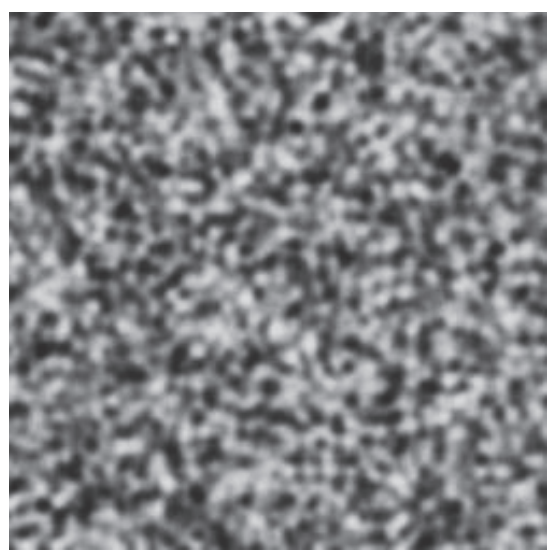

(a)

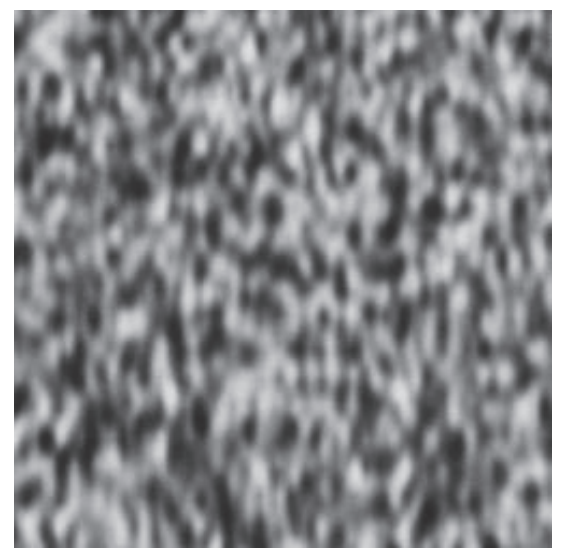

(b)

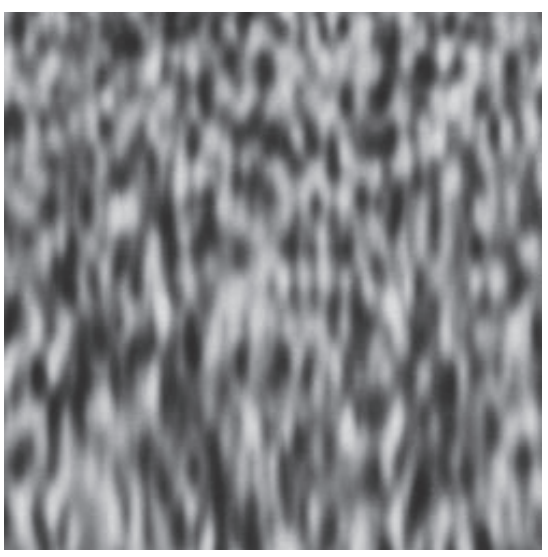

(c)

FIgURE 4: Rubber tensile images.

Based on the tension speed $v_{0}$, we can get the movement distance $x$ of material during exposure time and calculate the blur distance $y$ using the following formula:

$$
y=\frac{v_{0} t v}{u} .
$$

Using (9), we calculated the blur distance for different focal lengths and tensile rate list in Table 1.

As we can see from Table 1, the blur distance can reach $30 \mu \mathrm{m}$ and cause 5-pixel blur motions on images. Figure 4 shows the three images under different stretching conditions, the collected image will blur as the material is stretched, image details are lost, and edges become blurred. The blurred image significantly influences the accuracy of the video extensometer.

\section{Integer Pixel Displacement Search Based on Local Phase Quantization Features}

In order to get more accurately measured result, we use N-R iteration algorithm, but the convergent range of $\mathrm{N}$ - $\mathrm{R}$ iteration algorithm is only a few pixels [10]; in order to make the algorithm converge rapidly and accurately, an accurate initial estimate of the deformation parameter must be provided.
The conventional method for initializing the deformation parameter is using integer pixel displacement search. It is used to find the peak position of the correlation coefficient in a deformed image pixel by pixel. However, this method depends on gray information; it cannot handle large rotation and motion blur, because it assumes the subset shape is unchanged [11-16]. Recent works [17, 18] use the optimized parameter of the current point as the initial estimate for its neighbors based on the assumption that the deformations of neighboring points are very similar. The calculation started from a seed point manually selected in the undeformed image. The seed point's deformation parameter is initialized by using either manually specified correspondence or conventional search scheme. After being optimized, the parameter is transferred to an adjacent point. The whole procedure was repeated until all the data points have been analyzed. However, it still has several drawbacks. The series of images requires that the shape of its local region remains unchanged in the entire deformation process. This assumption is easily violated in large motion blur and deformation.

It can be seen that image blur will change the image information and make it difficult to identify the data point, thus affecting the video extensometer measurement accuracy. In order to make the video extensometer more accurate, we need to eliminate the influence of motion blur on integer 
TABLE 1: The blur distance for various types of lenses and tensile rate.

\begin{tabular}{|c|c|c|c|c|c|c|c|}
\hline $\begin{array}{l}\text { Focal length } \\
(\mathrm{mm})\end{array}$ & $\begin{array}{c}\text { Image } \\
\text { distance } \\
(\mathrm{mm})\end{array}$ & $\begin{array}{l}\text { Size of pixel } \\
(\mu \mathrm{m})\end{array}$ & $\begin{array}{l}\text { Exposure } \\
\text { time }(\mathrm{s})\end{array}$ & $\begin{array}{l}\text { Tensile rate } \\
(\mathrm{mm} / \mathrm{min})\end{array}$ & $\begin{array}{c}\text { Object } \\
\text { distance } \\
(\mathrm{mm})\end{array}$ & $\begin{array}{l}\text { Blur distance } \\
\qquad(\mu \mathrm{m})\end{array}$ & $\begin{array}{l}\text { Blur pixel } \\
\text { (pix) }\end{array}$ \\
\hline 20 & 21.42 & 5.2 & 0.01 & 100 & 300 & 1.19 & 0.23 \\
\hline 50 & 60 & 5.2 & 0.01 & 100 & 300 & 3.33 & 0.64 \\
\hline 80 & 109.09 & 5.2 & 0.01 & 100 & 300 & 6.06 & 1.17 \\
\hline 20 & 21.42 & 5.2 & 0.01 & 200 & 300 & 2.38 & 0.46 \\
\hline 50 & 60 & 5.2 & 0.01 & 200 & 300 & 6.67 & 1.28 \\
\hline 80 & 109.09 & 5.2 & 0.01 & 200 & 300 & 12.12 & 2.33 \\
\hline 20 & 21.42 & 5.2 & 0.01 & 500 & 300 & 5.95 & 1.14 \\
\hline 50 & 60 & 5.2 & 0.01 & 500 & 300 & 16.66 & 3.21 \\
\hline 80 & 109.09 & 5.2 & 0.01 & 500 & 300 & 30.30 & 5.83 \\
\hline
\end{tabular}

pixel displacement search. For blurred images matching, conventional methods are based on a prior knowledge of the blurred image to restore a clear image [19-21] and then matching the data point in the clear image. However, image restoration is an ill-posed problem process. Restoration effect of the blurred image depends on the prior knowledge about the degradation process and the efficiency of the restoration algorithm. Actually, we can directly extract invariant features from blurred images [22] to design matching algorithm [23], which not only consumes less computation resource but also improves the accuracy and precision.

Based on the above ideas, this chapter proposes an integer pixel searching algorithm based on LPQ feature [24] which is invariant to centrally symmetric blur and uniform illumination changes.

4.1. Blur Invariance Using Fourier Transform Phase. In digital image processing, the discrete model for spatially shiftinvariant blurring of an ideal image $f(x, y)$ resulting in an observed image $g(x, y)$ can be expressed by a convolution, given by

$$
g(x, y)=f(x, y) * h(x, y)+n(x, y),
$$

where $h(x, y)$ is the point spread function (PSF) of the system, $n(x, y)$ is additive noise, and $*$ denotes $2 \mathrm{D}$ convolution.

The LPQ feature model is based on the following assumptions: (1) the noise pollution can be ignored; (2) the PSF is the centrally symmetric. Depending on the above assumption, (10) can be written as

$$
g(x, y)=f(x, y) * h(x, y) .
$$

In the Fourier domain, this corresponds to

$$
G(x, y)=F(x, y) * H(x, y),
$$

where $G(x, y), F(x, y)$, and $H(x, y)$ are the discrete Fourier transforms (DFT) of the observed image $g(x, y)$, the ideal image $f(x, y)$, and the point spread function $h(x, y)$. Equation (12) can be separated into the magnitude and phase parts, resulting in

$$
\begin{gathered}
|G(x, y)|=|F(x, y) \times H(x, y)| \\
\varphi_{g}(u, v)=\varphi_{f}(u, v)+\varphi_{h}(u, v) .
\end{gathered}
$$

Because the PSF $h(x, y)$ is centrally symmetric, namely, $h(x, y)=h(-x,-y)$, its Fourier transform is always realvalued, and as a consequence, its phase is only a two-valued function, given by

$$
\varphi_{h}(u, v)= \begin{cases}0 & \text { if } H(u, v) \geq 0 \\ \pi & \text { if } H(u, v)<0\end{cases}
$$

This means that

$$
\varphi_{g}(u, v)= \begin{cases}\varphi_{f}(u, v) & H(u, v)>0 \\ \varphi_{g}(u, v)+\pi & H(u, v)<0\end{cases}
$$

From the above equation, when $H(u, v) \geq 0$, the phase of the observed image $G(u, v)$ at the frequencies is invariant to centrally symmetric blur.

4.2. Extraction of LPQ Feature. The LPQ feature is based on the blur invariance property of the Fourier phase spectrum described in Section 4.1. It uses the local phase information extracted by the 2D DFT, computed over a rectangular neighborhood $N_{(x, y)}$. The 2D DFT on pixel $(x, y)$, denoted as $F_{(x, y)}(u, v)$, is defined by

$$
\begin{aligned}
& F_{(x, y)}(u, v) \\
& =\sum_{\left(x^{\prime}, y^{\prime}\right) \in N_{x, y}} f\left(x-x^{\prime}, y-y^{\prime}\right) e^{-\left(j 2 \pi u x^{\prime}+j 2 \pi u y^{\prime}\right)} \\
& =W_{u}^{T} f_{(x, y)},
\end{aligned}
$$

where $W_{u}$ is the basis vector of the 2D DFT at a frequency $u$ and $f_{(x, y)}$ is another vector containing all $M^{2}$ image samples from $N_{(x, y)}$.

In LPQ, only four frequency points are considered, such as $u_{1}=[0, \alpha]^{T}, u_{1}=[a, 0]^{T}, u_{1}=[a, \alpha]^{T}$, and $u_{1}=[a,-\alpha]^{T}$, where $a$ satisfies $H(\alpha, \alpha) \geq 0$. For each pixel position, this results in a vector

$$
\begin{aligned}
& F_{(x, y)}=\left[F\left(u_{1},(x, y)\right), F\left(u_{2},(x, y)\right), F\left(u_{3},(x, y)\right),\right. \\
& \left.\quad F\left(u_{4},(x, y)\right)\right] .
\end{aligned}
$$




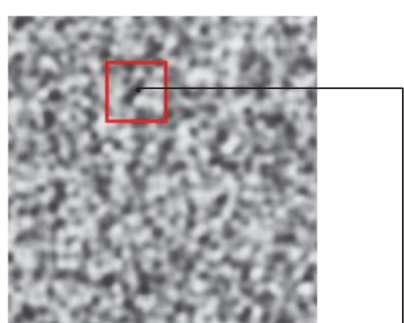

(a)

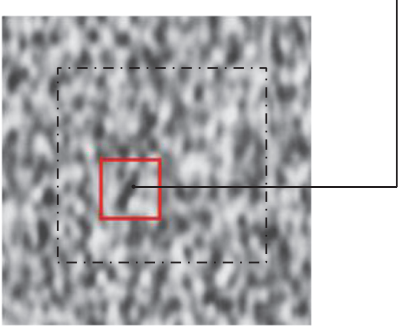

(b)

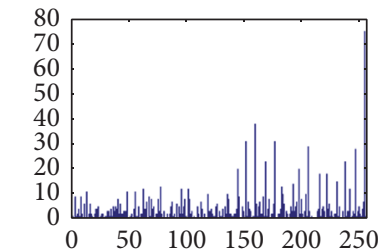

(c)

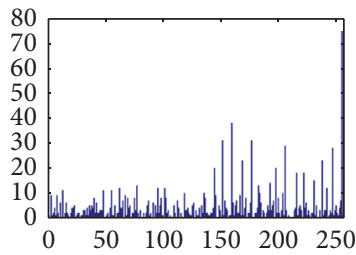

(d)

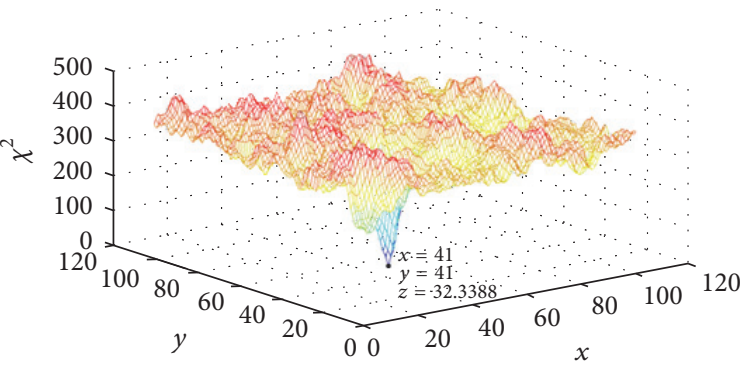

(e)

FIGURE 5: Flowchart for the algorithms routine: (a) undeformed image, (b) deformed image, (c) LPQ descriptor of reference subset, (d) LPQ descriptor of target subset, and (e) Chi-Square value.

The phase information in the Fourier coefficients is recorded by examining the signs of the real and imaginary parts of each component in $F_{(x, y)}$. This is done by using a simple scalar quantization:

$$
e_{i}= \begin{cases}1 & \text { if } g_{i} \geq 0 \\ 0 & \text { if } g_{i}<0,\end{cases}
$$

where $g_{i}$ is the $i$ th component of the vector $G_{(x, y)}=\left[\operatorname{Re}\left\{F_{x}\right\}\right.$, $\left.\operatorname{Im}\left\{F_{x}\right\}\right]$. The resulting eight binary coefficients $e_{i}$ are represented as integer values within $0-255$ using binary coding:

$$
f_{\mathrm{LPQ}}(x, y)=\sum_{i=1}^{8} e_{i}(x, y) 2^{i-1} .
$$

Finally, a histogram is formed by all the positions in the rectangular region and used as a 256-dimensional feature vector in the match.

4.3. Matching Based on the LPQ Feature. In this section, we introduce the key procedure of the matching algorithm. First, input a series of images, select the first one as the un-deformed image, and then select a data point $P$ on it. Secondly, a rectangular $M \times M$ neighborhood at the data point $P$ is selected as the reference subset, as shown in Figure 5(a). The LPQ descriptor of the reference subset is computed according to the method of the previous section, as shown in Figure 5(c). Thirdly, a region bigger than the reference subset area centered at the location of corresponding $P^{\prime}$ is defined as the searching subset on the deformed images. Then, we divide searching subset into subimages which have the same size as the reference subset and calculate the LPQ descriptor for each subimage, as shown in Figure 5(d). Finally, calculate the similarity between the reference subset and the rectangular regions of searching subset. Since the LPQ descriptor is a histogram, we can use the histogram distance calculation method to measure image similarity. The common histogram distance calculation methods are histogram intersection method, Bhattacharyya distance method, and Chi-Square statistics method. In this paper, we use Chi-Square statistics as the similarity metric. It is defined as follows:

$$
\chi^{2}(S, C)=\sum_{j} \omega_{j} \frac{\left(S_{j}-C_{j}\right)^{2}}{S_{j}+C_{j}},
$$

where $S$ and $C$ are the histograms of reference subset and rectangular regions, respectively. $S_{j}$ are $C_{j}$ are the $j$ th subregion of the histogram of the reference subset and the rectangular regions. $\omega_{j}$ is the weight values of the $j$ th subregion.

According to Chi-Square statistics, the location of the minimum value of $\chi^{2}(S, C)$, as shown in Figure 5(e), is the corresponding $P^{\prime}$ on the deformed image. The location $P^{\prime}$ is an initial estimate of the deformation parameter for the N-R iteration.

\section{Experimental Results}

In order to assess the performance of the initialization using LPQ matching, we performed uniaxial tensile experiments. In the DIC calculation, deformed images are generated by the bicubic spline interpolation. ZNSSD is selected as the objective function. The optimization is terminated if the change of ZNSSD is less than $10^{-8}$ or a maximum number of 20 iterations is reached. Unless specified, first-order shape function is used to model the deformation in the subset. 


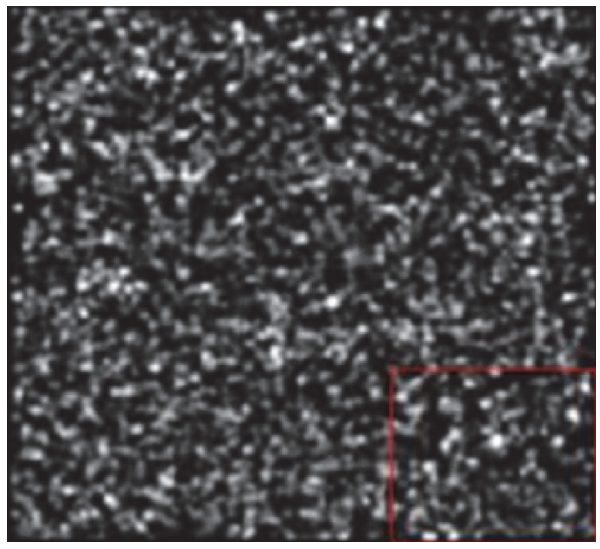

(a)

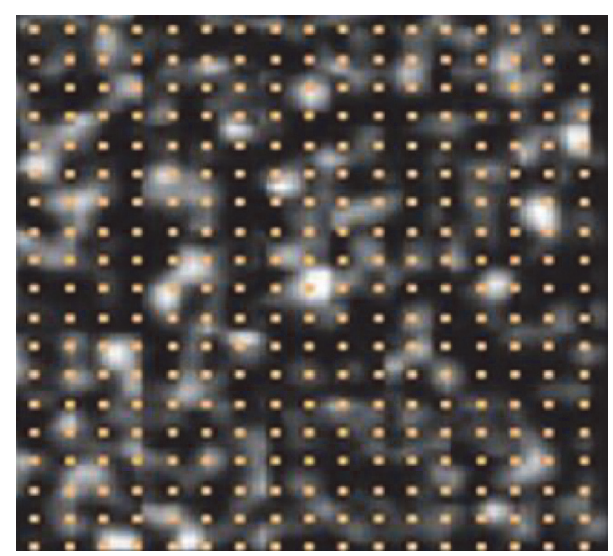

(b)

FIGURE 6: Simulate spackle pattern. (a) undeformed image; (b) the data point in the undeformed image.

5.1. Generation of Simulated Images. The simulated images are assumed to be the sum of individual Gaussian speckles:

$$
I(x, y)=\sum_{K=1}^{N} I_{k} \exp \left(\frac{\left(x-x_{k}\right)^{2}+\left(y-y_{k}\right)^{2}}{R^{2}}\right)
$$

where $N$ is the total number of speckles and $R$ is the speckle size. $x_{k}$ and $y_{k}$ are the positions of each speckle with a random distribution and $I_{k}$ is the peak intensity of each speckle. We use a speckle pattern with dimensions of $700 \times 700$ pixels, $N=1200$, and $R=4$ as the undeformed image, as shown in Figure 6(a).

5.2. Accuracy and Preassigned Displacement. In this experiment, a series of deformed images are generated using the bicubic spline interpolation method with a range of 0.1-0.9 pixels by step of 0.1 pixels between successive images. We use the first image as the undeformed image, as shown in Figure 6(a). Next, 50 data points are uniformly sampled in a rectangular region by a grid step of 5 pixels to measure the displacement of the other deformation images, as shown in Figure 6(b). The reference subset of each data point is $41 \times 41$. The measured mean error, maximum error, and standard deviation of the 50 data points selected in the 9 deformed images are calculated. The result of the conventional method [13] is listed in Table 2, and the result of proposed method is listed in Table 3; depending on the focal length, a pixel on the image is equivalent to $30 \mu \mathrm{m}$ on the material.

\subsection{Simulated Deformation Combined with Large Motion} Blur. To further illustrate the advantage of using LPQ feature in the initialization of DIC, we add the motion blur on the spackle pattern range from 0 to 10 pixels and step by 2 pixels. Considering when the materials fracture, the extension rate will increase rapidly and add the maximum fuzzy scale to 10 pixels as well. The proposed method is compared with the conventional method in the blurred spackle pattern. The mean error and the standard deviation of the two different methods on different motion blur are shown in Figures 7 and 8 , respectively.
TABLE 2: Results of the conventional method.

\begin{tabular}{lccc}
\hline Exact value & Mean error & Maximum error & Standard deviation \\
\hline 0.3 & 0.005421 & 0.007142 & 0.006942 \\
0.6 & 0.004254 & 0.016541 & 0.005987 \\
0.9 & 0.004852 & 0.023214 & 0.008532 \\
1.2 & 0.004024 & 0.019654 & 0.008974 \\
1.5 & 0.006104 & 0.012100 & 0.012746 \\
1.8 & 0.005845 & 0.008451 & 0.010924 \\
2.1 & 0.005112 & 0.013542 & 0.010572 \\
2.4 & 0.007521 & 0.016587 & 0.011348 \\
2.7 & 0.007310 & 0.018547 & 0.012679 \\
\hline
\end{tabular}

Unit: $\mu \mathrm{m}$.

TABLE 3: Results of proposed method.

\begin{tabular}{lccc}
\hline Exact value & Mean error & Maximum error & Standard deviation \\
\hline 0.3 & 0.002977 & 0.004726 & 0.005061 \\
0.6 & 0.001535 & 0.003642 & 0.005682 \\
0.9 & 0.001735 & 0.003623 & 0.007752 \\
1.2 & 0.001606 & 0.003372 & 0.008871 \\
1.5 & 0.001683 & 0.002956 & 0.010356 \\
1.8 & 0.002745 & 0.004856 & 0.010449 \\
2.1 & 0.003995 & 0.006735 & 0.008532 \\
2.4 & 0.002234 & 0.004235 & 0.007263 \\
2.7 & 0.003962 & 0.005275 & 0.005625 \\
\hline
\end{tabular}

Unit: $\mu \mathrm{m}$.

It can be analyzed that, with the increase of the motion blur, the mean error and the standard deviation of the conventional method increase; however, the accuracy of the proposed method is steady because of LPQ feature, but the conventional method cannot search the integral pixel displacement accurately when motion blur changes the image gray information. 


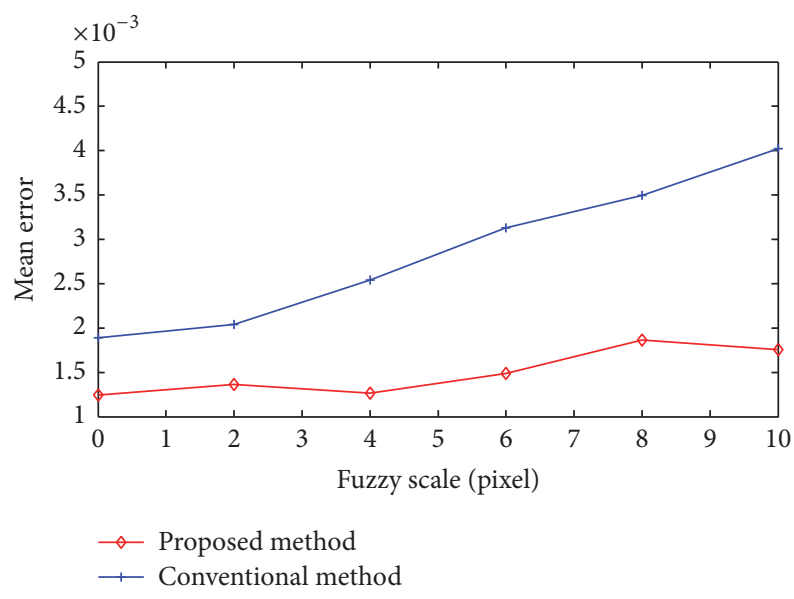

Figure 7: Comparison of mean error between the two search methods.

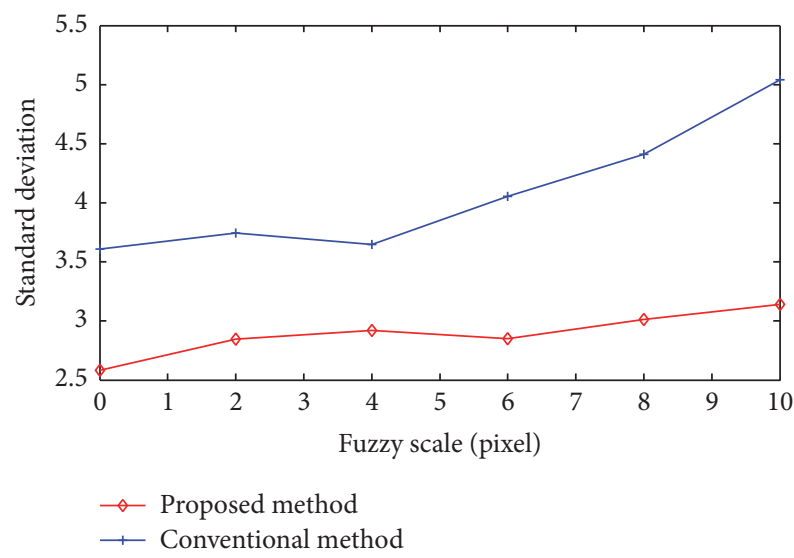

FIGURE 8: Comparison of standard deviation between the two search methods.

5.4. Simulated Deformations Combined with Different Size of Subset Region. The size of reference subset is an important parameter in DIC. When the size of subset region is smaller, computational efficiency is higher. However, less information contained in the smaller subset may reduce the match accuracy. If we increase the subset size, it can reduce the noise impact and get more accurate results. However, the calculation will increase proportionally with the subset size increase.

We choose reference subset size from $11 \times 11-71 \times 71$ pixels and then increase it by $20 \times 20$ pixels. The mean error for different reference subset size is shown in Figure 9. We can see that, with the reference subset increase in the size from $11 \times 11$ to $41 \times 41$ pixels, the precision increases, whereas it starts to increase slowly as the size exceeds $41 \times 41$ pixel. Here we chose the $41 \times 41$ pixels for the size of subset region.

5.5. Displacement Measurement of Uniaxial Tensile Experiment. In the presented video extensometer system, images are captured with Basler avA1000-120KM Camera Link analog monochrome cameras and a Matrox Meteor-II analog frame grabber card. The cameras are equipped with CCTV\&VIDEO

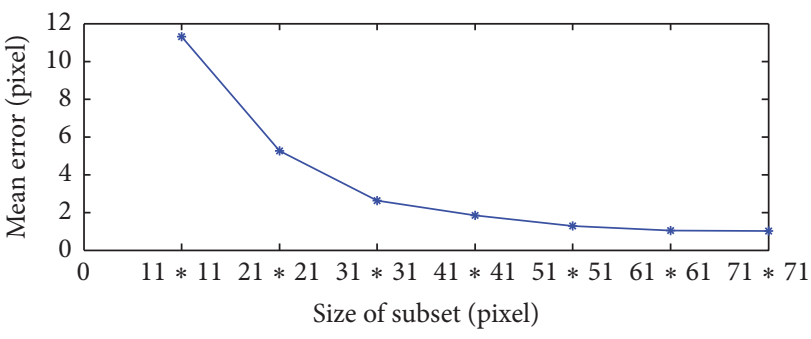

FIgURE 9: Comparison of standard deviation between different subset regions.

TABLE 4: Results of proposed method.

\begin{tabular}{lcc}
\hline Number & Average displacement & $C_{\text {znssd }}$ \\
\hline 1 & $225.00,90.26$ & 0.0092 \\
2 & $225.00,89.22$ & 0.0218 \\
3 & $225.12,85.36$ & 0.0081 \\
4 & $225.56,71.51$ & 0.0116 \\
5 & $225.48,19.55$ & 0.0209 \\
\hline
\end{tabular}

TABLE 5: Results of the conventional method.

\begin{tabular}{lcc}
\hline Number & Average displacement & $C_{\text {znssd }}$ \\
\hline 1 & $225.00,90.86$ & 0.0160 \\
2 & $225.00,89.04$ & 0.0320 \\
3 & $225.20,84.52$ & 0.0360 \\
4 & $225.96,70.98$ & 0.0680 \\
5 & $225.58,18.34$ & 0.0940 \\
\hline
\end{tabular}

LENS AVENIR ZOOM LENS ST16160 lenses with focal length of $16-160 \mathrm{~mm}$.

The shape of the high elongation specimen typically used for uniaxial tensile tests follows the GB/T 528-2009 standard. The width of the specimen is $5.0 \pm 1.0 \mathrm{~mm}$; thickness of the specimen is $2.0 \pm 0.2 \mathrm{~m}$.

During the experiment, we use the first image as the undeformed image and other 5 images at different times as the deformed images. On the undeformed image, we choose 50 data points on the same line and calculate its displacement. The average displacement and average ZNSSD values for these data points at different deformed images are listed in Table 4. Meanwhile, the measurements of conventional method are listed in Table 5.

In Table 4 it is shown that the average displacement is similar in the first and second deformed images but after the third picture, images appear with large deformation and motion blur; the ZNSSD of conventional method increase significantly, up to 0.094 , at the last deformed images. The results of the proposed method are still accurate and the ZNSSD does not have obvious change. Also the displacement of conventional method is larger than that of the proposed method on the $y$-axis which is stretching direction. 


\section{Discussion and Conclusion}

In this paper, we propose a novel integer pixel searching algorithm based on LPQ feature utilized in the video extensometer system that can match the data points during the uniaxial tensile testing.

Experimental results on simulated speckle images verify that this algorithm is better than conventional methods, the maximum mean error is only $0.003995 \mu \mathrm{m}$, and the relative error is $1.33 \%$ which satisfies the accuracy of extensometer. Then, we add motion blur on the speckle image; experimental results showed that accuracy of the proposed method will not be affected by the motion blur. The impact on accuracy that the size of subset region has is also studied; we find that, considering both accuracy and efficiency, the size of $41 \times 41$ a pixels was the optimal option. Finally, the proposed method is tested in the deformation measurement of a high elongation specimen. The experiment results show that the proposed method has excellent performance in the actual application.

In conclusion, the algorithm can bring a more accurate and more intelligent measurement technique for measuring full-field displacements of high elongation materials.

\section{Competing Interests}

The authors declare that they have no competing interests.

\section{Acknowledgments}

The authors would like to thank Xiaofang Yuan for his constructive suggestions. This work is supported by the National Natural Science Foundation of China (Grant no. 61501181), by the National Science and Technology Major Project of China (Grant no. 07-Y30B10-9001-14/16), by the Priority Academic Program Development of Jiangsu Higher Education Institutions, and by Jiangsu Collaborative Innovation Center on Atmospheric Environment and Equipment Technology.

\section{References}

[1] C. Yueting, "Primary discussion on measuring technology in video extensometer," Engineering \& Test, vol. 9, pp. 50-53, 2009.

[2] B. Pan, L. Yu, and D. F. Wu, "High-accuracy 2D digital image correlation measurements with bilateral telecentric lenses: error analysis and experimental verification," Experimental Mechanics, vol. 53, no. 9, pp. 1719-1733, 2013.

[3] B. Pan, L. Yu, D. Wu, and L. Tang, "Systematic errors in twodimensional digital image correlation due to lens distortion," Optics and Lasers in Engineering, vol. 51, no. 2, pp. 140-147, 2013.

[4] B. Pan, L. Tian, and X. L. Song, "Real-time, non-contact and targetless measurement of vertical deflection of bridges using off-axis digital image correlation," NDT \& E International, vol. 79, pp. 73-80, 2016.

[5] B. Pan, K. Qian, H. Xie, and A. Asundi, "Two-dimensional digital image correlation for in-plane displacement and strain measurement: a review," Measurement Science and Technology, vol. 20, no. 6, Article ID 062001, 2009.
[6] B. Pan, H. Xie, Z. Guo, and T. Hua, "Full-field strain measurement using a two-dimensional Savitzky-Golay digital differentiator in digital image correlation," Optical Engineering, vol. 46, no. 3, Article ID 033601, 2007.

[7] B. Gu, V. S. Sheng, Z. Wang, D. Ho, S. Osman, and S. Li, "Incremental learning for $v$-Support Vector Regression," Neural Networks, vol. 67, pp. 140-150, 2015.

[8] B. Pan, H. Xie, Y. Xia, and Q. Wang, "Large-deformation measurement based on reliable initial guess in digital image correlation method," Acta Optica Sinica, vol. 29, no. 2, pp. 400-406, 2009.

[9] W. Tong, "An evaluation of digital image correlation criteria for strain mapping applications," Strain, vol. 41, no. 4, pp. 167-175, 2005.

[10] M. A. Sutton, C. Mingqi, W. H. Peters, Y. Chao, and S. McNeill, "Application of an optimized digital correlation method to planar deformation analysis," Image \& Vision Computing, vol. 4, no. 3, pp. 143-150, 1986.

[11] B. Gu, V. S. Sheng, K. Y. Tay, W. Romano, and S. Li, "Incremental support vector learning for ordinal regression," IEEE Transactions on Neural Networks \& Learning Systems, vol. 26, no. 7, pp. 1403-1416, 2015.

[12] F. Hild, B. Raka, M. Baudequin, S. Roux, and F. Cantelaube, "Multiscale displacement field measurements of compressed mineral-wool samples by digital image correlation," Applied Optics, vol. 41, no. 32, pp. 6815-6828, 2002.

[13] Z.-F. Zhang, Y.-L. Kang, H.-W. Wang, Q.-H. Qin, Y. Qiu, and X.-Q. Li, "A novel coarse-fine search scheme for digital image correlation method," Measurement, vol. 39, no. 8, pp. 710-718, 2006.

[14] B. Pan, F. W. Da, and X. Yong, "Incremental calculation for large deformation measurement using reliability-guided digital image correlation," Optics and Lasers in Engineering, vol. 50, no. 4, pp. 586-592, 2012.

[15] J. Li, X. Li, B. Yang, and X. Sun, "Segmentation-based image copy-move forgery detection scheme," IEEE Transactions on Information Forensics and Security, vol. 10, no. 3, pp. 507-518, 2015.

[16] B. Pan, "Reliability-guided digital image correlation for image deformation measurement," Applied Optics, vol. 48, no. 8, pp. $1535-1542,2009$.

[17] B. Pan, Z. Wang, and Z. Lu, "Genuine full-field deformation measurement of an object with complex shape using reliabilityguided digital image correlation," Optics Express, vol. 18, no. 2, pp. 1011-1023, 2010.

[18] H. Cheong, E. Chae, E. Lee, G. Jo, and J. Paik, "Fast image restoration for spatially varying defocus blur of imaging sensor," Sensors, vol. 15, no. 1, pp. 880-898, 2015.

[19] B. Chen, H. Shu, G. Coatrieux, G. Chen, X. Sun, and J. L. Coatrieux, "Color image analysis by quaternion-type moments," Journal of Mathematical Imaging and Vision, vol. 51, no. 1, pp. 124-144, 2015.

[20] M. R. Banham and A. K. Katsaggelos, "Digital image restoration," IEEE Signal Processing Magazine, vol. 14, no. 2, pp. 24-41, 1997.

[21] Y. Peng, T. Wu, S. Wang, N. Kwok, and Z. Peng, "Motionblurred particle image restoration for on-line wear monitoring," Sensors, vol. 15, no. 4, pp. 8173-8191, 2015.

[22] Z. Xia, X. Wang, X. Sun, and B. Wang, "Steganalysis of least significant bit matching using multi-order differences," Security and Communication Networks, vol. 7, no. 8, pp. 1283-1291, 2014. 
[23] X. Wen, L. Shao, Y. Xue, and W. Fang, "A rapid learning algorithm for vehicle classification," Information Sciences, vol. 295, no. 1, pp. 395-406, 2015.

[24] V. Ojansivu, E. Rahtu, and J. Heikkila, "Rotation invariant local phase quantization for blurinsensitive texture analysis," in Proceedings of the 19th International Conference on Pattern Recognition (ICPR '08), pp. 3330-4005, Tampa, Fla, USA, 2008. 


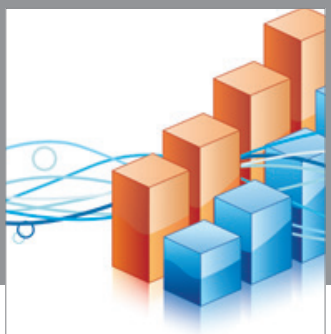

Advances in

Operations Research

vatem alat4

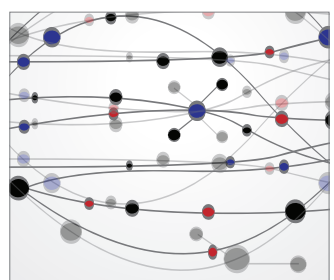

\section{The Scientific} World Journal
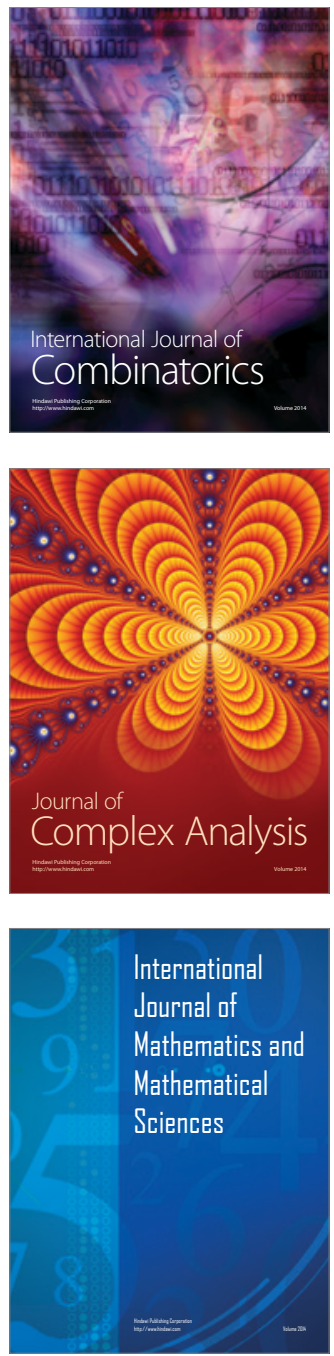
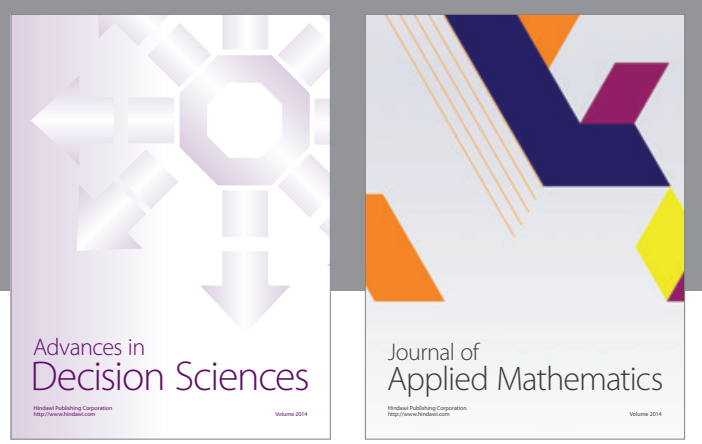

Algebra

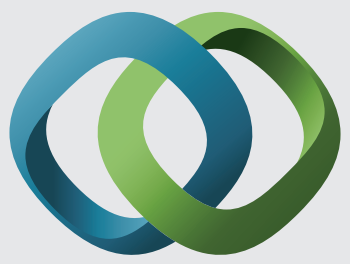

\section{Hindawi}

Submit your manuscripts at

http://www.hindawi.com
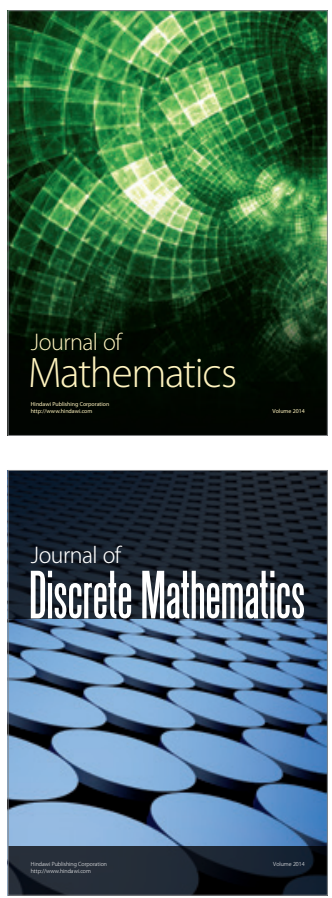

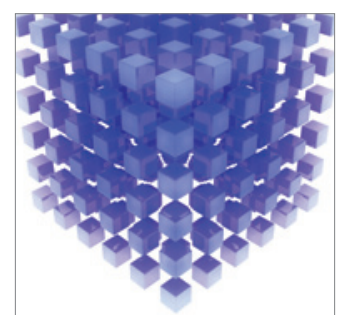

Mathematical Problems in Engineering
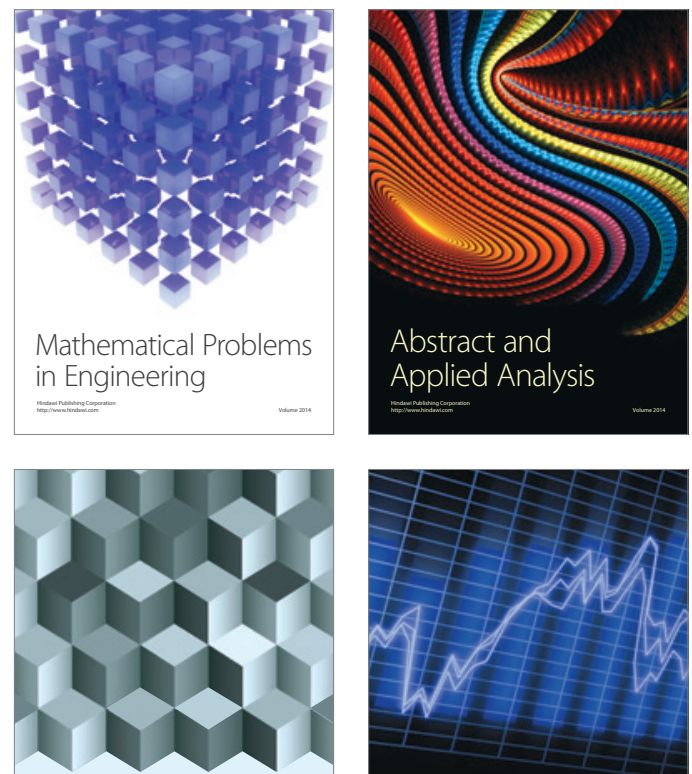

Journal of

Function Spaces

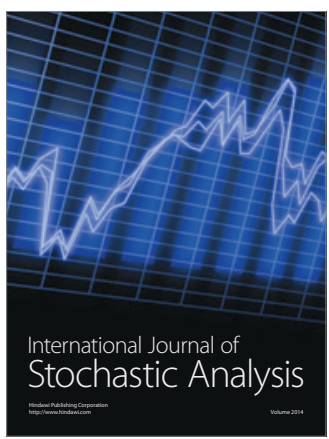

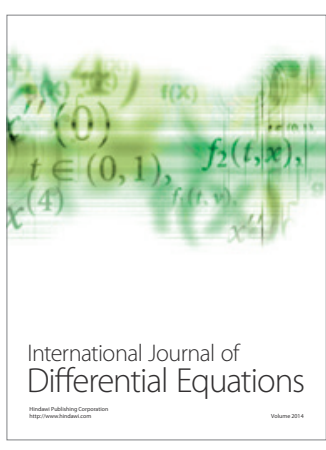
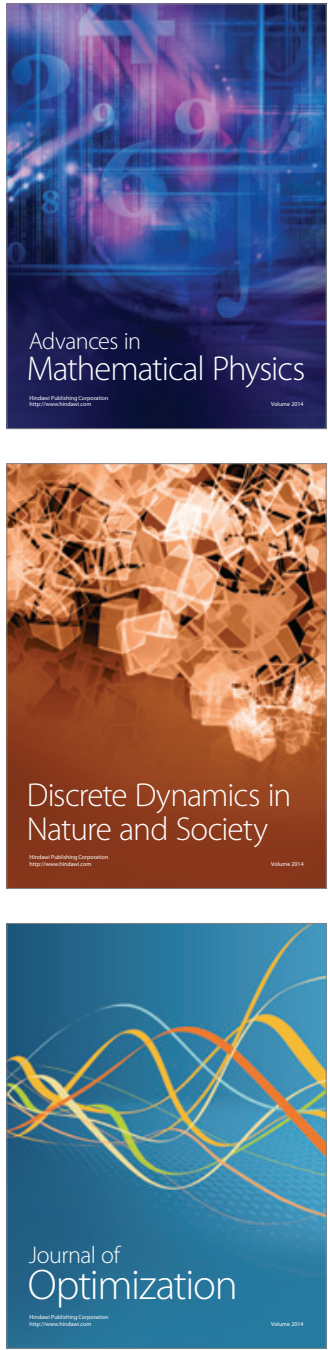